\title{
RNA Duplex Recognition and Unwinding by DEAD-Box Helicase DDX3X
}

\author{
$X \mathrm{Ji}^{1}, \mathrm{H}$ Song ${ }^{1}, \mathrm{~L} \mathrm{Jin}^{1}, \mathrm{~A} \mathrm{Ram}^{1}$ \\ ${ }^{1}$ National Cancer Institute, NIH, Frederick, MD \\ jix@mail.nih.gov
}

DEAD-box helicases (DDXs) regulate RNA processing and metabolism by unwinding short double-stranded (ds) RNAs. Sharing a helicase core composed of two RecA-like domains (D1D2), DDXs function in an ATPdependent, non-processive manner. As an attractive target for cancer and AIDS treatment, DDX3X and its orthologs are extensively studied, yielding a wealth of biochemical and biophysical data, including structures of apo-D1D2 and post-unwound D1D2:single-stranded RNA complex, and the structure of a D2:dsRNA complex that is thought to represent a pre-unwound state. However, the structure of a pre-unwound D1D2:dsRNA complex remains elusive, and thus, the mechanism of DDX action is not fully understood. Here, we describe the structure of a D1D2 core in complex with a 23-base pair dsRNA at pre-unwound state, revealing that two DDXs recognize a 2turn dsRNA, each DDX mainly recognizes a single RNA strand, and conformational changes induced by ATP binding unwinds the RNA duplex in a cooperative manner. Human DDX3X and its yeast ortholog Ded1p represent a subfamily of DDXs. The worldwide effort in structural and mechanistic analysis of these two DDXs demonstrated that they function as a trimer wherein the protomers play distinct loading and unwinding roles, but the exact number of protomers involved in each role is not clear. Based on new structural and functional data, we conclude that one protomer is responsible for loading and the other two are responsible for unwinding the RNA duplex.

Acta Cryst. (2020). A76, a50 